\title{
VALEUR PATRIMONIALE DE SERRASALMINAE HERBIVORES DU HAUT MARONI (GUYANE FRANÇAISE) : APPROCHES BIOLOGIQUE ET SOCIOCULTURELLE EN PAYS WAYANA.
}

\author{
H. PAGEZY (1), M. JÉGU (2)
}

(1) Unité d'Eco-anthropologie, FRE 2323 du CNRS, Muséum National d'Histoire Naturelle, 45 rue Cuvier, 75231 PARIS Cedex 05. E-mail : hpagezy@ club-internet.fr (corresponding author).

(2) Antenne IRD, Laboratoire d'Ichtyologie, Muséum National d'Histoire Naturelle, 43 rue Cuvier, 75231 PARIS Cedex 05. E-mail : jegu@mnhn.fr

\section{RÉSUMÉ}

La valeur patrimoniale des kumaru, espèces de poissons appartenant à la sousfamille des Serrasalminae, a été approchée selon des critères faisant référence à leur place au sein de la biodiversité ou de la sociodiversité. Du point de vue de l'anthropologue, les kumaru apparaissent indéniablement comme les espèces de poissons ayant globalement les plus fortes valeurs sociale, hédonique, économique, non seulement chez les Amérindiens Wayana du Haut Maroni, mais aussi chez les Noirs Boni du cours moyen. On peut les qualifier à ce titre d'espèces patrimoniales. Du point de vue du biologiste, les trois espèces de kumaru du Haut Maroni se situent à des niveaux d'endémicité différents, mais toutes participent au fonctionnement du biotope particulier des herbiers à Podostemaceae, au moins dans leurs jeunes stades. Au-delà de la simple conservation des espèces, le mode de distribution de l'asitau (Prosomyleus rhomboidalis) et la position du watau yaikë (Tometes lebaili) dans la phylogénie des Serrasalminae leur confèrent un ensemble de qualités entrant dans l'évaluation de leur valeur patrimoniale. L'habitat de ces espèces, milieu fragile et très particulier, participe aussi à la patrimonialité.

Mots-clés : valeur patrimoniale, Serrasalminae, Guyane française, endémicité, écologie, socioanthropologie, Indiens Wayana. 


\title{
PATRIMONIAL VALUE OF HERBIVOROUS SERRASALMINAE FISH OF THE UPPER MARONI STREAM (FRENCH GUYANA): BIOLOGICAL AND SOCIO-CULTURAL APPROACHES IN THE WAYANA REGION.
}

\begin{abstract}
The patrimonial value of kumaru, 3 species of fish belonging to Serrasalminae, has been approached from 2 perspectives: their importance in biodiversity and their value in sociodiversity. From the anthropological point of view, the kumaru appear to be the fish species with the highest social, hedonic and economic value, not only among the Amerindians living by the upper Maroni river, but also among the Boni of the lower Maroni. For these reasons, they may be considered as patrimonial resources. From the biologist's point of view, the three kumaru species of the upper Maroni show different levels of endemicity, but they all contribute towards the particular biotope of the Podostemaceae herb, at least during their younger stages. Beyond the simple care for species conservation, the distribution of the asitau and the place of the watau yaikë among the phylogeny of the Serrasalminae, confer on them a set of qualities contributing to the estimation of their patrimonial value. The habitat of these species, a very unstable biotope, also contribute to their value as patrimonial resources.
\end{abstract}

Key-words : patrimonial value, Serrasalminae, French Guyana, endemicity, ecology, socioanthropology, Wayana Indians.

\section{INTRODUCTION}

Dans son acceptation première, la qualification de «patrimoniale » renvoie à la transmission de biens familiaux d'une génération à l'autre. D'origine juridique, ce terme, qui associe patrimoine et héritage, a connu certaines extensions de sens, en particulier en relation avec les « objets vivants " que représentent les milieux naturels et les espèces sauvages, animales ou végétales. C'est en 1972 que la conférence générale de l'UNESCO adopte la «Convention du Patrimoine mondial » qui a pour mission de dresser la « ... liste des sites dont les valeurs exceptionnelles doivent être préservées ... ". Le patrimoine naturel apparaît dans nos sociétés fortement lié aux préoccupations de protection (préservation ou conservation) de la nature, de mise en valeur de la biodiversité, de gestion à long terme des ressources naturelles (CORMIER-SALEM et ROUSSEL, 2000).

Parmi les critères associés à la notion d'espèce patrimoniale exprimés par une population parisienne (BOUTHELOUP, 1999), apparaissent d'abord ceux d'espèce menacée, en danger, d'espèce symbolique ou mythique, puis d'espèce sympathique (ou antipathique), d'espèce endémique ou à distribution réduite alors que les notions d'intérêt écologique ou économique apparaissent moins souvent dans cette étude.

Les dix critères de détermination des espèces patrimoniales, tels que présentés par la DIREN (1998) pour l'élaboration de la liste des espèces patrimoniales de Guyane française, permettent de mieux cerner cette notion chez les scientifiques. L'endémisme, la rareté naturelle, une aire de distribution limitée, un habitat restreint ou menacé et enfin la raréfaction par pression sur l'habitat ou sur le stock, constituent les sept critères écologiques retenus. Ces critères sont tous liés à la notion de conservation d'un taxon potentiellement en danger. Deux autres critères tiennent compte de la relation entre l'homme et le taxon considéré ; ils font référence à sa valeur culturelle ou pédagogique et à sa valeur agronomique ou économique. Enfin, la position phylogénétique particulière du taxon introduit un dixième critère faisant référence à l'évolution et à la paléohistoire dans la notion de patrimonialité. 
Ainsi, au regard des biologistes, une espèce endémique - originaire d'une région et caractéristique d'un milieu - appartient presque de plein droit au patrimoine naturel, d'autant plus qu'elle est considérée comme menacée. Son génome participe à l'enrichissement de la diversité génétique du milieu tandis que sa valeur patrimoniale peut s'en trouver fortement accrue lorsque celle-ci, devenue rare, n'existe plus que sur une aire limitée. La notion de patrimoine naturel est ainsi associée à la territorialité : appliquée au contexte aquatique, marin ou dulçaquicole, l'aire de répartition d'une espèce peut se limiter à la totalité ou seulement à une partie de cours d'eau, à une mangrove ou aux grands fonds abyssaux. La disparition de cette espèce peut être révélatrice de la perturbation du milieu, suite à la pression d'espèces exogènes à la dynamique plus agressive, ou à l'action destructrice de l'Homme. Transmis de génération en génération au cours de millénaires voire de millions d'années, le patrimoine génétique confère à certaines espèces une valeur exceptionnelle aux yeux des scientifiques : c'est le cas du coelacanthe, poisson quasiment mythique, qualifié de "fossile vivant ", ayant trouvé refuge dans les grands fonds de l'Océan Indien, et qui déchaîne des passions chez les biologistes de l'évolution et les paléontologistes (JANVIER, 1999).

Qu'il s'agisse d'une espèce ou d'un milieu, la valeur biologique du patrimoine naturel repose sur sa participation à la diversité biologique, dimension qui déborde largement le contexte local. La science étant universelle, on peut par extension parler de patrimoine national voire mondial.

Il est une autre façon d'envisager la valeur patrimoniale d'une espèce vivante. Celleci fait référence à la valeur que lui attribue l'Homme, en particulier les sociétés vivant à son contact, qui la connaissent, l'utilisent et lui attribuent une place privilégiée dans leur système de représentation. Ainsi, le statut patrimonial d'une espèce ne pourrait être dissocié des « savoirs et pratiques qui lui sont associés et des logiques sociales mises en œuvre (d'identité, de différentiation, de relation à la nature, au lieu, au passé, de modes d'appropriation et de règles d'utilisation) " (CORMIER-SALEM, 2000).

En bref, tant du point de vue du biologiste que de l'anthropologue, le concept de patrimoine naturel sous-entend que l'on attribue à une espèce ou un milieu naturel une certaine valeur, définie par sa place au sein de l'écosystème (rareté, endémisme par exemple) ou au sein de la société (valeur identitaire, valeur d'usage par exemple).

Nous nous proposons d'examiner la valeur patrimoniale de Serrasalminae phytophages, poissons herbivores ou frugivores, inféodés aux hauts cours des fleuves qui drainent le plateau guyanais, et plus particulièrement au haut cours du Maroni, à la frontière de la Guyane française et du Surinam.

Limitée en aval par Maripasoula, petit centre administratif, l'aire concernée par notre réflexion s'étend vers le sud, région habitée par les Wayana, population amérindienne installée sur les berges de la Litany (villages de Taluène, Twenké, Antecume Pata, Péléa et Pidima) (Figure 1). Taluène et Twenké sont situés sur une portion très calme du fleuve, alors que les autres villages, plus en amont, sont proches de zones très accidentées et torrentueuses. Depuis Maripasoula, l'accès aux deux premiers villages en pirogue à moteur est assez aisé quelle que soit la saison. En revanche, la navigation vers l'amont, déjà difficile pendant la crue, requiert une connaissance parfaite du fleuve pendant l'étiage. A cette saison, le transport entre Maripasoula et Antecume Pata dure plus d'une demijournée pendant l'étiage. 


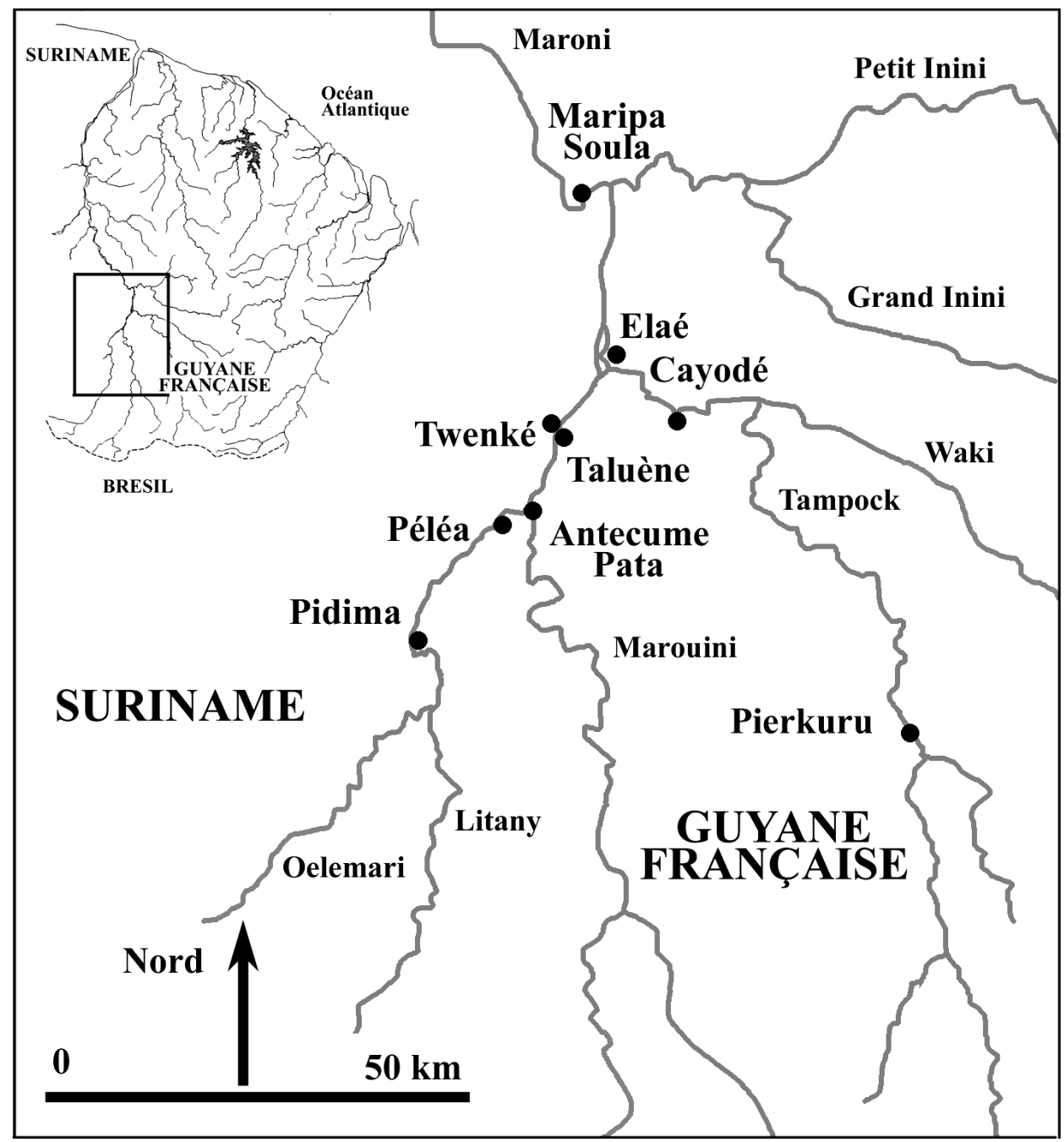

Figure 1

Localisation géographique de la zone d'étude.

Figure 1

The geographical placement of the study.

\section{LES KUMARU}

Les trois espèces, qui font l'objet de cette réflexion, appartiennent à la sous-famille des Serrasalminae. Elles sont regroupées dans la région sous le vocable de koumarou (kumaru, kumalu, coumarou), mais ont leur propre dénomination dans le vocabulaire wayana' ${ }^{1}$ il s'agit de watau ihle (Mylopus n. sp.), watau yaikë (Tometes lebaili) et asitau (Prosomyleus rhomboidalis).

\footnotetext{
${ }^{1}$ Dans la suite du texte, le terme kumaru sera employé pour désigner l'ensemble des deux watau et de l'asitau.
} 
Les informations orales obtenues sur les pratiques de la pêche dans la région de Taluène indiquent que les kumaru sont très rarement capturés dans les zones calmes. En effet, les deux espèces de watau, tout comme l'asitau, sont particulièrement inféodées aux rapides, mais ne fréquentent pas les criques. Par ailleurs, aucune capture, ni aucune technique visant à capturer ces poissons, que ce soit dans les criques ou en forêt inondée, ne nous a été signalée.

L'asitau, Prosomyleus rhomboidalis, est une espèce strictement rhéophile, présente dans tous les hauts cours qui drainent le plateau guyanais, fleuves côtiers, affluents de l'Amazone ou de l'Orénoque, et dans les rapides du Rio Xingu sur le plateau Central brésilien (Figure 2a). Seule espèce de Serrasalminae qui soit pan-guyanaise, elle est caractérisée par des mâchoires fortes, armées de dents molariformes lui permettant d'écraser les graines les plus résistantes. En effet, les graines constituent la majeure partie de son alimentation; elles peuvent être remplacées en saison sèche par des Podostemaceae (BOUJARD et al., 1990). Les juvéniles sont carnivores et se nourrissent de petits invertébrés présents entre les feuilles des herbiers à Podostemaceae, dans les rapides et les sauts. Selon LECOMTE et al. (1993), les plus grands spécimens de $P$. rhomboidalis seraient âgés de 5 ans. Dans le bassin du Maroni l'asitau peut peser $3,5 \mathrm{~kg}$ et mesurer près de $40 \mathrm{~cm}$ de long.

Le watau yaikë est une espèce du genre Tometes (Figure 2b), citée comme Myleus pacu par PLANQUETTE et al. (1996) et décrite par JÉGU et al. (2002) dans ce même volume. Elle est strictement rhéophile, s'alimente surtout de feuilles et de fruits de Podostemaceae, mais peut aussi se nourrir pendant l'étiage de résidus ligneux, et au moment de la crue de fruits de la végétation rivulaire (JÉGU et PAGEZY, 1999). Comme pour Prosomyleus rhomboidalis, les juvéniles de cette espèce sont présents dans les rapides et les sauts entre les feuilles des herbiers à Podostemaceae. Aujourd'hui, le Tometes lebaili n'est connu que du haut cours du Maroni, de la Mana et du Commewine, bien qu'un spécimen semble provenir du bas cours de la Sinnamary, sans que sa provenance puisse être absolument certifiée. Les individus de cette espèce peuvent atteindre $5,0 \mathrm{~kg}$.

Le watau ihle est l'espèce la plus grande, pouvant atteindre $5,8 \mathrm{~kg}$ (obs. pers. : saut Pierkuru, riv. Tampok, oct. 2000). II s'agit d'une espèce nouvelle pour la science (Figure 2c), du genre Myloplus, endémique du haut cours du Maroni. Jusqu'à nos récentes observations, ce poisson n'était connu des scientifiques que par des spécimens de grande taille, capturés au cours de pêches traditionnelles à la nivrée dans les grands sauts, ou aux filets maillants dans les portions rocheuses de cours plus calmes. Les mâchoires et les dents de Mylopus n. sp. sont moins fortes que celles de Prosomyleus rhomboidalis. Nos premières observations indiquent que Mylopus $\mathrm{n}$. sp. s'alimente de fruits, fleurs et feuilles de la végétation rivulaire. Le watau ihle est plus rare que les deux espèces précédentes dans les rapides : au cours de pêches traditionnelles à la nivrée en octobre 2000 , nous n'avons récolté qu'un juvénile, alors que nous récoltions de nombreux juvéniles d'asitau et de watau yaikë.

Avec l'aimara (Hoplias aimara, espèce qui " pourrait atteindre $40 \mathrm{~kg}$ " selon PLANQUETTE et al., 1996), les deux espèces d'huluwi (Pseudoplatystoma fasciatum et P. tigrinum atteignant 15 à $17 \mathrm{~kg}$ selon LE BAIL et al., 2000), le matawale (Cichla ocellaris atteignant $10 \mathrm{~kg}$ selon SANTOS et al., 1984) et le pëne (Serrasalmus rhombeus : plus de $3 \mathrm{~kg}$ selon PLANQUETTE et al., 1995), toutes carnivores, les watau et l'asitau font partie des plus grosses espèces de poissons que l'on trouve dans le haut Maroni. 


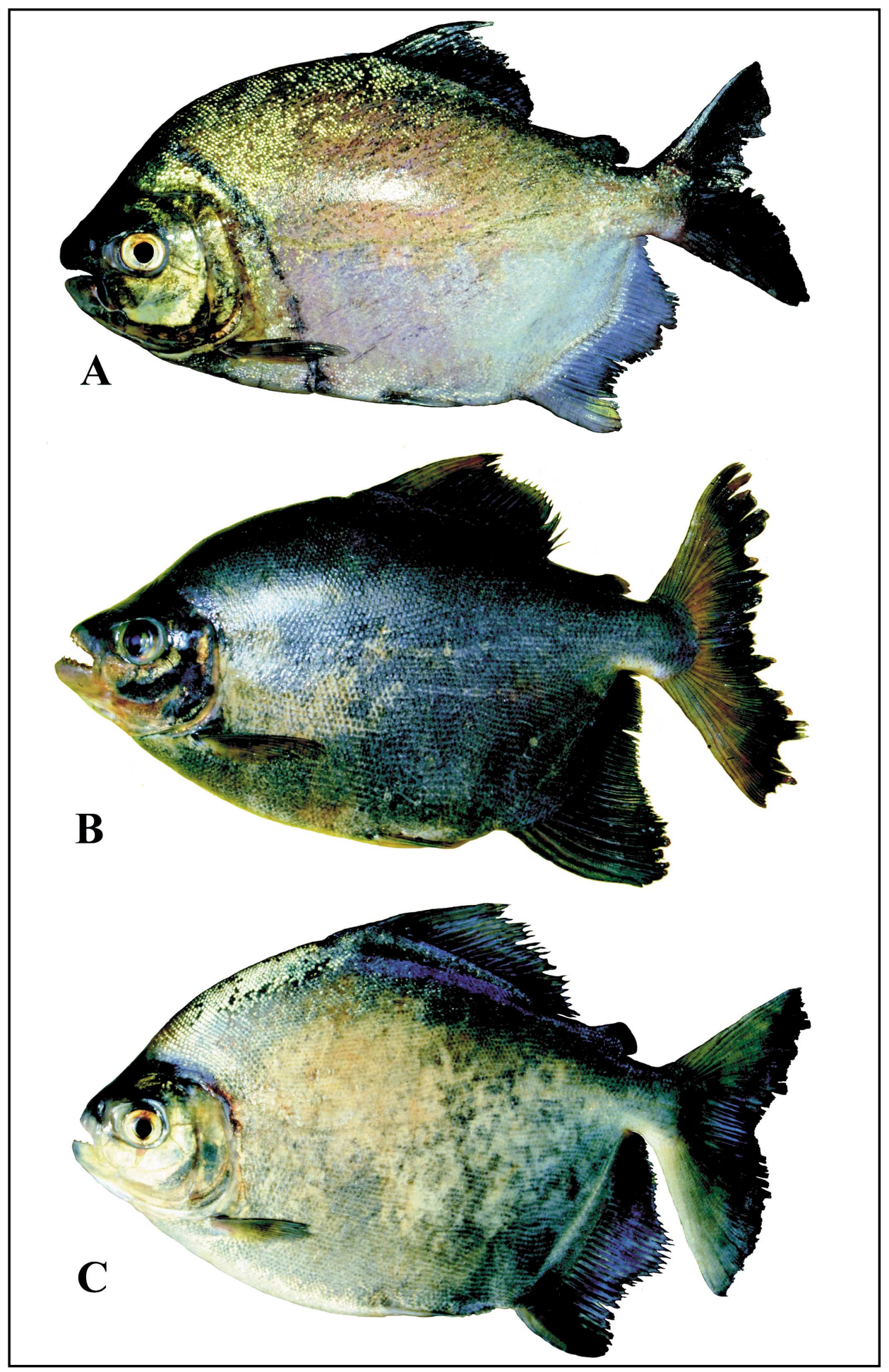

Figure 2

Les kumaru du Haut Maroni : A - Prosomyleus rhomboidalis, asitau ; B - Tometes lebaili, watau yaikë ; C - Mylopus sp., watau ihle (photos M. JÉGU).

\section{Figure 2}

The kumaru fishes of Upper maroni: A - Prosomyleus rhomboidalis, asitau; B - Tometes lebaili, watau yaikë; C - Mylopus sp., watau ihle (photos by M. JÉGU). 


\section{VALEUR PATRIMONIALE DES GRANDS SERRASALMINAE HERBIVORES AU REGARD DES BIOLOGISTES}

A partir des 10 critères retenus par la DIREN (1998, cf. supra), KEITH et al. (2000) proposent que cinq espèces de Serrasalminae soient classées comme patrimoniales pour la Guyane française, dont trois espèces endémiques : le watau ilhe (Mylopus sp.) et le watau yaikë (Tometes lebaill) du Maroni, le Tometes trilobatus de l'Oyapock.

Par ailleurs, les scientifiques ont retenu d'autres critères importants pour justifier de la qualité patrimoniale du watau yaikë ${ }^{2}$ et de l'asitau. Ceux-ci font référence à l'habitat tout à fait particulier de ces espèces, ainsi qu'à leurs particularités biogéographiques et phylogéographiques.

\section{Les kumaru, espèces associées aux herbiers à Podostemaceae}

La présence de kumaru dans les hauts cours des fleuves qui drainent le bouclier guyanais est étroitement liée à celle des herbiers à Podostemaceae. Les Podostemaceae sont des dicotylédones aquatiques rupestres, vivant dans les zones à très fort courant (Figure 3). Les feuilles du Mourera fluviatilis (wija ou «peau du caillou » des Wayana), espèce la plus répandue sur le Haut Maroni, peuvent atteindre plusieurs dizaines de centimètres de diamètre.

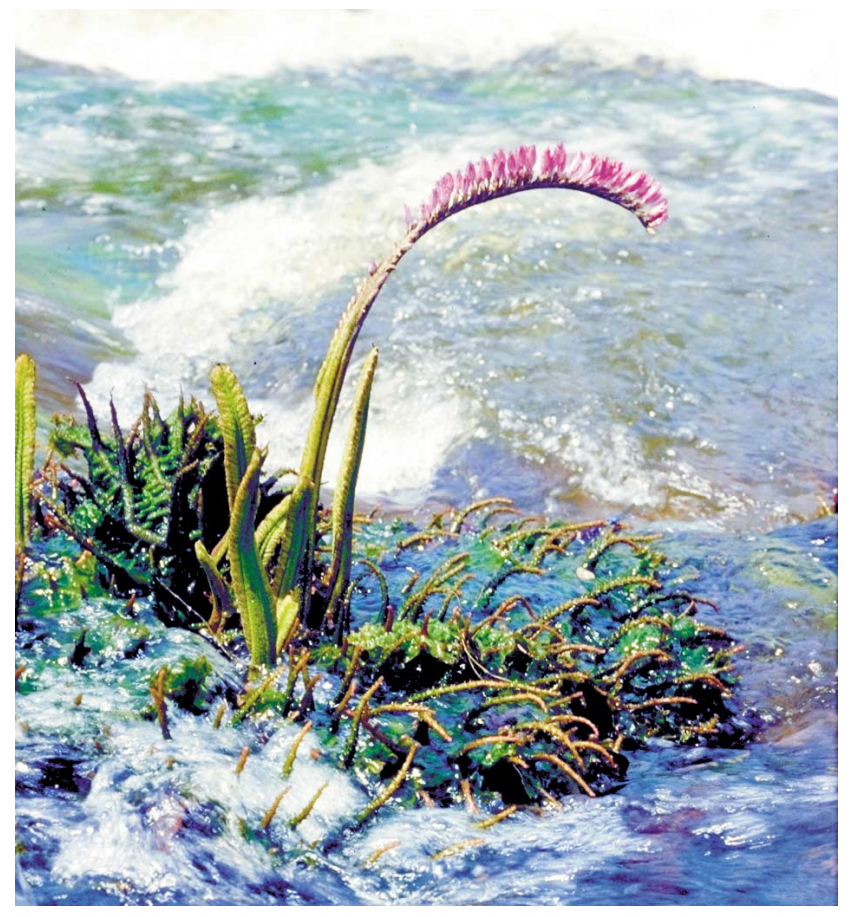

Figure 3

Les herbiers à Podostemaceae en fleur (wiija) dans une portion torrentueuse du haut cours d'un fleuve du plateau des Guyanes (photo M. JÉGU).

\section{Figure 3}

Flowering Podostemaceae (wija) in an upstream section of rapids on the Guyanas plateau (photo by M. JÉGU).

\footnotetext{
${ }^{2}$ Les critères de patrimonialité retenus pour le watau ilhe ont uniquement trait à sa condition d'endémisme par manque de connaissance scientifique sur cette espèce.
} 
Une faune particulière est associée aux Podostemaceae, constituée entre autres de larves d'insectes, d'arachnides, de micro-crustacés, de planaires et de sangsues qui s'abritent entre leurs feuilles (ODINETZ-COLLARD et al., 1996). Ces herbiers ont aussi un rôle de nurserie pour les jeunes kumaru qui y trouvent refuge et s'alimentent d'invertébrés. Les adultes de Tometes (watau yaikë) et Mylesinus (pacu cana au Brésil), genre proche du précédent et présent sur le versant sud du bouclier guyanais, s'alimentent presque exclusivement de Podostemaceae (SANTOS et al., 1997). En revanche, l'asitau, phytophage et frugivore, a un régime alimentaire beaucoup plus diversifié (BOUJARD et al., 1990 ; FERREIRA, 1992). La lumière de l'intestin, chez les adultes de Mylesinus (THATCHER et JÉGU, 1996) ou de Tometes (obs. pers.), contient plusieurs milliers de nématodes Rondonia rondoni ainsi que des centaines de trématodes amphistomes. Cet hyperparasitisme, qui ne provoque aucune pathologie évidente de l'hôte, est considéré par certains auteurs comme un endo-commensalisme suggérant une fonction destinée à faciliter la digestion des Podostemaceae (ODINETZ-COLLARD et al., 1996). La présence de ces invertébrés dans les rapides, en introduisant des niveaux trophiques supplémentaires, permet d'accroître la diversité fonctionnelle de ces fleuves très pauvres en éléments nutritifs (GOULDING et al., 1988).

Les herbiers à Podostemaceae sont des milieux très sensibles aux actions anthropiques ayant pour conséquence la mise en suspension de sédiments provoquée par la prospection minière (MEUNIER et al., 1998, Figure 11e) ou l'altération du cycle hydrologique en aval des barrages. La disparition de ces biotopes peut mettre en danger, non seulement les espèces de poissons qui, comme le watau yaikë, sont strictement inféodés aux Podostemaceae (ODINETZ-COLLARD et al., 1996), mais aussi la dynamique de l'écosystème au sein duquel vivent les amérindiens. C'est une des raisons pour lesquelles HOFF (2000) place les groupements à Mourera fluviatilis, comme les autres groupements à Podostemaceae, dans la liste des habitats patrimoniaux pour la Guyane française.

\section{Singularités biogéographiques et phylogéographiques des kumaru}

Parce qu'ils sont endémiques de certains pays, voire de certains cours d'eau du bassin amazonien, les kumaru représentent une richesse en tant que patrimoines biologique et génétique. Cette richesse renvoie à la dynamique évolutive locale, en particulier à la microévolution liée à l'histoire du bassin amazonien. Pour VIVIEN (2000), il s'agit d'une valeur " d'usage indirect » qui correspond à la "valeur écologique de la diversité biologique, c'est à dire son rôle fonctionnel, tant dans l'évolution des espèces que dans la dynamique des écosystèmes ».

\section{L'asitau}

L'asitau (Prosomyleus rhomboidalis) est une espèce abondante sur tout le pourtour du bouclier guyanais, à l'exception du haut Rio Negro, de l'Orénoque et ses affluents (JÉGU, obs. pers.). La distribution de cette espèce rhéophile euryendémique (MYERS et GRAVE, 2000) est restreinte aux hauts cours des fleuves, mais elle s'étend aussi sur les bas cours des fleuves côtiers, zones sous influence de forts courants de marée. Etant donnée la fragmentation de l'aire de distribution de $P$. rhomboidalis en une mosaïque de populations disjointes, et dans le cas où l'histoire des gènes serait restée fidèle à l'histoire de ses populations, cette espèce représente un témoin privilégié des paléo-évènements qui ont participé à la mise en place du biotope auquel elle est associée. On peut la considérer à ce titre comme un élément du patrimoine historique. 


\section{Le watau yaikë}

Lorsqu'on considère les relations phylogénétiques au sein des Serrasalminae (PORTO et al., 1991 ; ORTI et al., 1996) les Tometes et les Mylesinus représentent un rameau frère de l'asitau, l'ensemble constituant un rameau frère des piranhas (Figure 4).

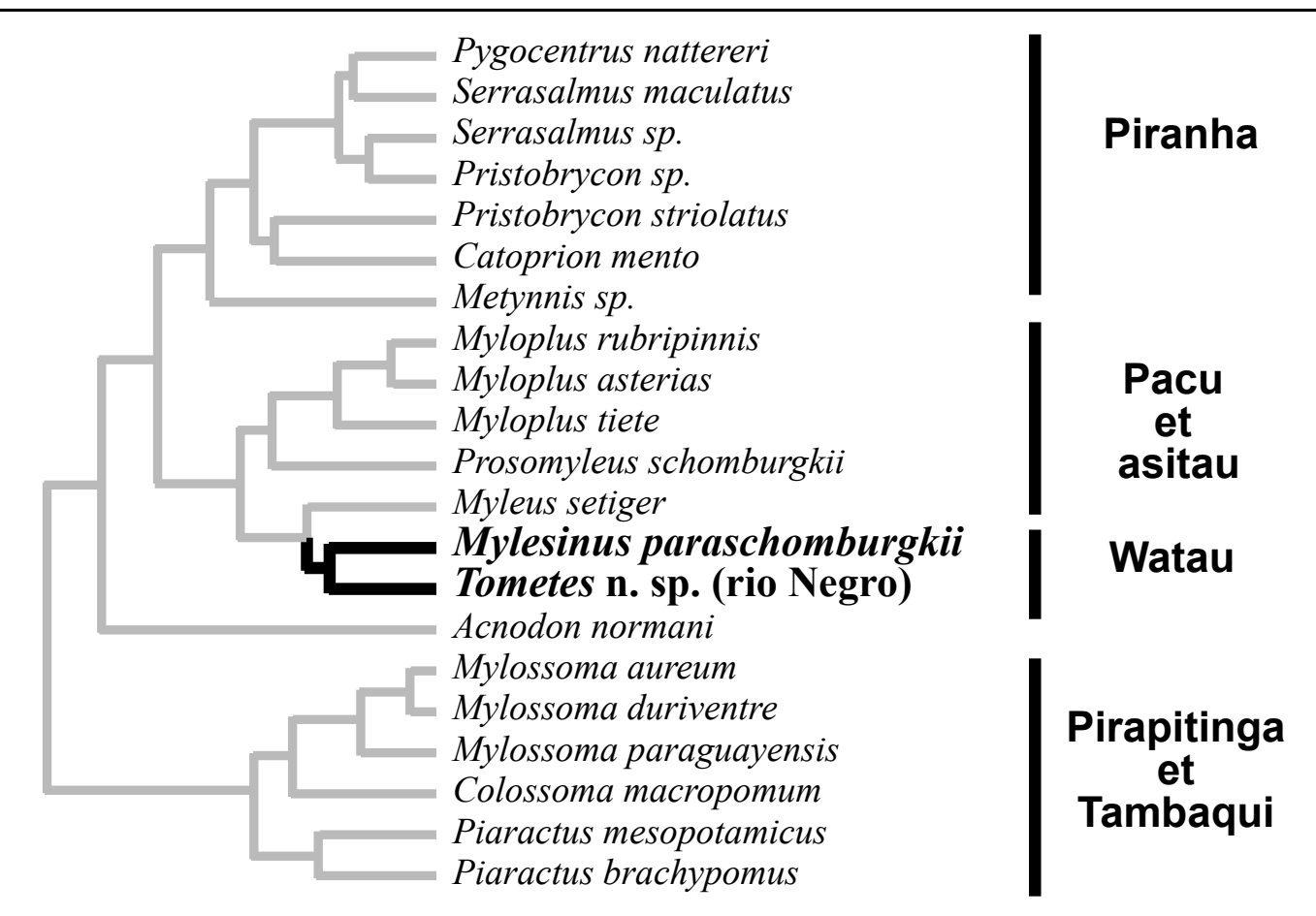

Figure 4

Phylogénie des Serrasalminae (Characidae) modifié d'après ORTI et al. (1995).

\section{Figure 4}

The phylogeny of the Serrasalminae (Characidae) subspecies, modified from ORTI et al. (1995).

Le Tometes (watau yaikë) et le Mylesinus (pacu cana) sont des espèces strictement inféodées aux Podostemaceae. Ces deux taxa sont dispersés sur les boucliers guyanais et brésilien, suivant une distribution disjointe qui rappelle celle de l'asitau. Mais dans ce cas, il s'agit de deux genres dont les espèces présentent un endémisme d'étendue beaucoup plus limitée. En effet, la distribution de Mylesinus schomburgkii est limitée à l'Essequibo (Guyana), alors que M. paucisquamatus est endémique du bassin du Tocantins (Brésil) et l'aire de distribution de M. paraschomburgkii est située entre l'Uatumã et l'Araguari, sur le versant sud du bouclier Guyanais (Figure 5). Dans le genre Tometes, l'espèce trilobatus est connue de l'Araguari à l'Oyapock, tandis que le Tometes lebaili (watau yaikë) se limite à la Mana, au Maroni et au Commewine. En outre, une nouvelle espèce de Tometes, dont la distribution se limite au Rio Negro et à l'Essequibo, est en cours de description. 


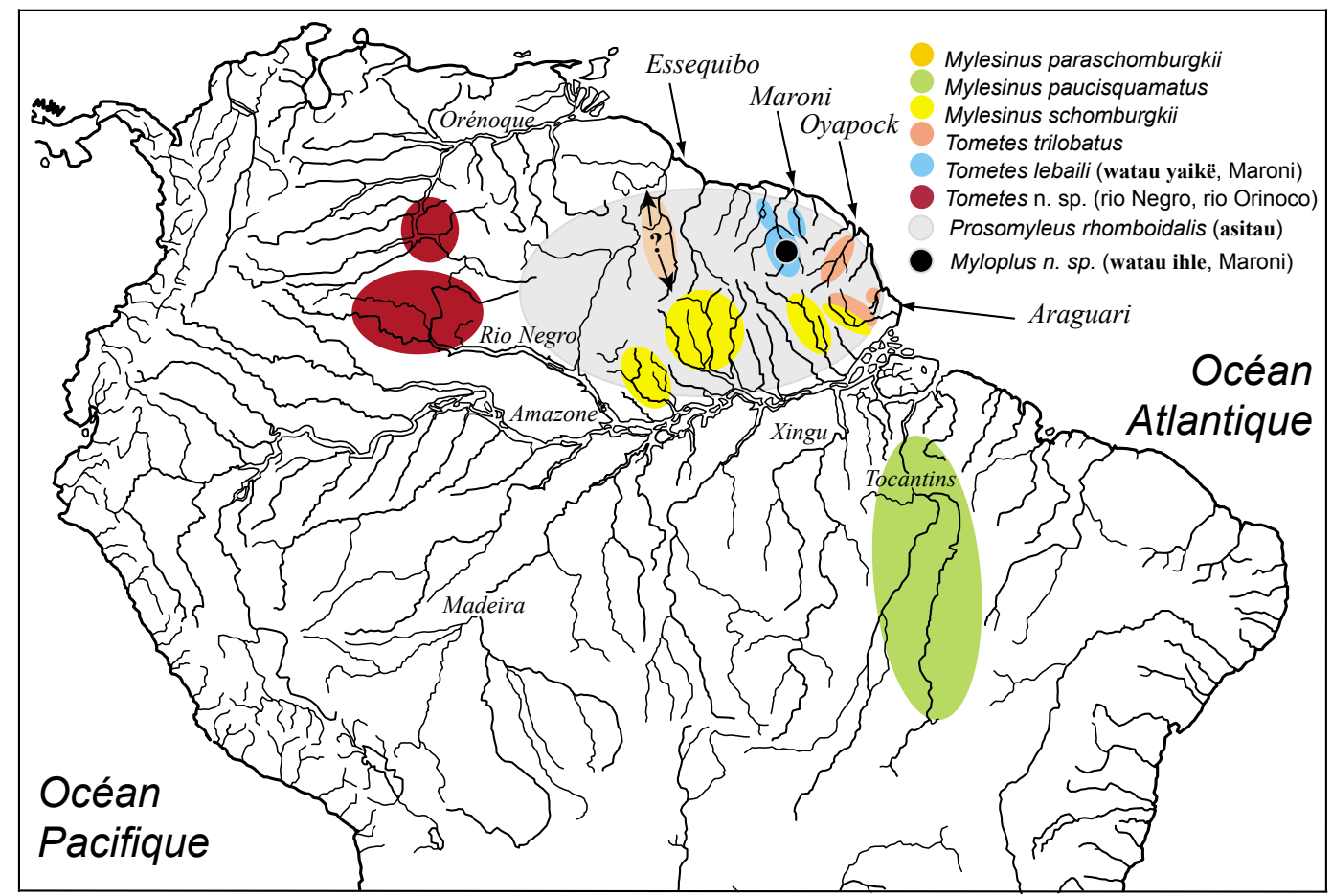

Figure 5

Distribution des espèces de Tometes et Mylesinus (Serrasalminae, Characidae) dans la partie septentrionale de l'Amérique du Sud.

\section{Figure 5}

The distribution of the species Tometes and Mylesinus (Serrasalminae, Characidae) in the northern part of South America.

L'interprétation biogéographique de l'endémisme dépend de son niveau taxonomique. Ainsi, les Mylesinus et Tometes figurent parmi les taxa les plus dérivés des Serrasalminae. II s'agit d'espèces spécialisées dont la distribution s'est trouvée limitée par un certain nombre de contraintes exercées par le milieu.

Aborder la complexité de la mise en place de ces endémismes par l'étude des relations phylogénétiques des espèces permet d'accéder à la connaissance des évènements paléo-écologiques ayant pu marquer les relations entre les bassins qui en sont le siège. Les informations contenues dans le matériel génétique du watau yaikë, comme dans celui des autres Tometes et Mylesinus, prennent alors une dimension bien plus large. II ne s'agit plus simplement de modèles de l'évolution, mais bien de pages de l'histoire du bassin amazonien qui appartiennent au patrimoine mondial.

\section{VALEUR PATRIMONIALE DES GRANDS SERRASALMINAE HERBIVORES POUR LES INDIENS WAYANA}

La qualification de patrimoniale d'une espèce sous entend qu'une société lui attribue une importante valeur sociale. Cette valeur, de nature culturelle ou économique, apparaît d'abord comme une valeur d'usage (VIVIEN, 2000) qui, transposée aux kumaru, est essentiellement alimentaire. 


\section{Valeur des kumaru en tant qu'aliments}

\section{Valeur symbolique}

Repris par de nombreux auteurs, LEVI STRAUSS $(1962,128)$ a avancé que les espèces naturelles n'ont pas été choisies parce que "bonnes à manger mais parce que bonnes à penser ». Ainsi, les Wayana classent les aliments en bons ou mauvais, selon la " force » qui leur ont été attribuée et dont ils incorporent les propriétés en les ingérant (FISCHLER, 1989). Cette " force " est d'autant plus puissante et dangereuse que la teneur en " sang " que le créateur Kujuli leur a donnée lorsqu'il a créé le monde est élevée (CHAPUIS, 1998). Les hommes et les puissances surnaturelles, premiers servis par le Créateur, qui ne résultent d'aucune transformation, donc n'ont été le siège d'aucune dilution, sont aussi les plus dangereux ; puis vient le gibier, en particulier les espèces de grande taille ${ }^{3}$, riches en sang (foncé) leur conférant une grande force vitale (omole), susceptible d'accélérer le vieillissement des personnes qui les consomment mais nécessaires aussi à revitaliser leur organisme. En revanche, les poissons, les végétaux, et les éléments inertes comme les criques - cours d'eau secondaires - qui ont reçu du créateur un " sang " affaibli par les dilutions successives au cours de processus de transformations, sont peu dangereux pour l'organisme. $\mathrm{Si}$, d'une façon générale, les poissons sont considérés comme de "bons aliments " ou " aliments authentiques " (tëhem mïle), sans danger pour la santé, les petits poissons à écailles opi (Characidae), le pasina blanc (Paramyloplus ternetzi, Serrasalminae) et la carpe talani (Leporinus friderici, Anostomidae), seules espèces à ne pas provenir d'une transformation de chenilles, représentent la pureté absolue. Parmi les Serrasalminae, seuls les poissons carnivores comme le piranha (pëne) représentent un danger potentiel par la qualité de leur sang, alors que les autres Serrasalminae, herbivores, seraient totalement (pasina blanc) ou presque totalement (watau) inoffensives. Ainsi, sans toutefois représenter la pureté absolue, les watau qui nous intéressent, sont considérés par les Wayana comme de «bons aliments ».

Compte tenu des qualités intrinsèques des poissons en général, il n'est pas étonnant de constater leur importance dans le régime alimentaire des Wayana : de $260 \mathrm{~g}$ à $370 \mathrm{~g}$ par jour et par adulte (et jusqu'à $600 \mathrm{~g}$ pour certains hommes adultes) selon le sexe et l'âge (FRERY et al, 1999) ; de $268 \mathrm{~g}$ à $390 \mathrm{~g}$ par personne et par jour, jusqu'à $692 \mathrm{~g}$ par pêcheur et par jour au cours d'une nivrée commerciale (JÉGU et PAGEZY, 1999). Ces résultats mettent en évidence l'adéquation entre valeur symbolique du poisson et son importance dans le régime alimentaire des Indiens, les quantités énormes ingérées quotidiennement ayant pour conséquence la satisfaction des besoins nutritifs en protéines de bonne qualité.

\section{Valeur hédonique}

Les kumaru sont des espèces particulièrement appréciées des Wayana. Ces poissons rehaussent la qualité d'un repas, assurent du prestige d'un présent, avant l'huluwi (Pseudoplatystoma) et souvent au même titre que l'aimala (Hoplias aimara), deux poissons également de grande taille mais carnivores ${ }^{4}$.

Chez les Wayana, le gras et la fermeté de la chair sont des qualités fort prisées. Elles sont reconnues aux watau, surtout en saison des pluies. Les Wayana expliquent la

\footnotetext{
${ }^{3}$ Bien que la puissance hématologique ne dépende pas que de la taille, certains animaux plus menaçants auraient un sang plus puissant que d'autres espèces de plus grande taille mais considérées comme moins dangereuses.

${ }^{4}$ La préférence pour les kumaru sur l'aimala semble être plus marquée dans les villages du cours supérieur (Pidima, Antecume), peut être à cause de la plus grande proximité avec ces espèces.
} 
plus forte teneur en graisse de ces espèces par leur changement de régime alimentaire ${ }^{5}$. La tête du poisson, à cause de la graisse que l'on peut voir au fond de la bouche ou dans la cavité orbitale, est considérée comme un morceau de choix car c'est là que se loge tout le parfum de l'animal. Néanmoins, certaines personnes avouent ressentir parfois une lassitude suite à une consommation trop importante de watau, reprochant aux individus trop gras de générer dégoût et somnolence.

Les kumaru sont des poissons très recherchés, non seulement des Indiens wayana, mais aussi des Noirs marrons, installés en aval de Maripasoula sur le Maroni. Leur comportement de hâte au moment des arrivages de kumaru à Maripasoula témoigne dans le même sens. Comme chez les Indiens ce sont les espèces les plus appréciées, celles qu'ils sont disposés à payer le prix fort. Jaloux des bons morceaux, les Boni reconnaissent accorder difficilement à leur enfant la tête d'un kumaru avant l'âge de 10 ans.

\section{Valeur patrimoniale des modes d'appropriation des kumaru}

La valeur patrimoniale des kumaru ne peut être séparée de la connaissance par les Amérindiens de la biologie et du comportement de ces poissons, et de la mise en jeu des principales techniques de production : la pêche à la nivrée en saison sèche et la pêche à la ligne appâtée de fruits en saison des pluies. A plusieurs reprises, nous avons pu constater la transmission précoce de ces techniques et de savoirs aux enfants wayana.

\section{La pêche à la nivrée}

La pêche à la nivrée, ou pêche au poison végétal, est la pêche de saison sèche par excellence ; elle est également connue des Boni des cours inférieurs (HURAULT, 1963). Individuelle, familiale ou villageoise, on l'organise lorsque l'étiage est au plus bas, du mois d'août au mois de décembre.

Cette technique consiste à épandre un principe ichtyotoxique (roténone) (GRENAND et MORETTI, 1982), contenu dans la sève des lianes hali hali (Lonchocarpus chrysophyllus Kleinh, Fabacae) dans des portions de rivières parfois assez vastes, au niveau des rapides (sauts), dans les petits cours d'eau (criques) ou dans des endroits marécageux de forêt (pripri).

Une pêche à la nivrée débute par le battage des lianes qui, une fois écrasées, sont placées dans des katuri (hottes en feuilles de palmier fraîchement coupées), brassées et lessivées dans le courant. Seules les grandes nivrées, qu'elles soient villageoises ou commerciales, ont pour cible préférentielle les kumaru, à la différence des nivrées plus modestes, pratiquées en eau vive dans les sauts, les bras morts ou les marécages.

II est remarquable de noter que cette technique de pêche est dotée d'une valeur identitaire faisant référence à un mythe wayana ${ }^{6}$ selon lequel la liane hali hali, descendue à terre, se transforme en jeune Amérindien (Figure 6), puis s'intègre au groupe en y prenant femme afin de se révéler à ces derniers.

Les grandes nivrées villageoises, qui rassemblent un ou plusieurs villages, totalisent un nombre très élevé de pirogues et de pêcheurs : 24 pirogues pour la nivrée à laquelle nous avons assisté en octobre 2000 au saut Pierkuru sur le Tampok ${ }^{7}$, soit quelque 50 hommes adultes ${ }^{8}$.

\footnotetext{
${ }^{5}$ En saison des pluies, les watau recherchent les nombreuses espèces de fruits dont ils sont friands et qui abondent et tombent le long des rives.

${ }^{6}$ Récits de Panapasi et de Kulyaman, Antecume Pata, octobre 2000.

${ }^{7}$ Les Wayana évoquent de grandes nivrées communautaires rassemblant 2 à 3 fois plus de pirogues.

${ }^{8}$ Femmes et enfants non inclus, les pirogues comptant chacune de 5 à 11 personnes.
} 


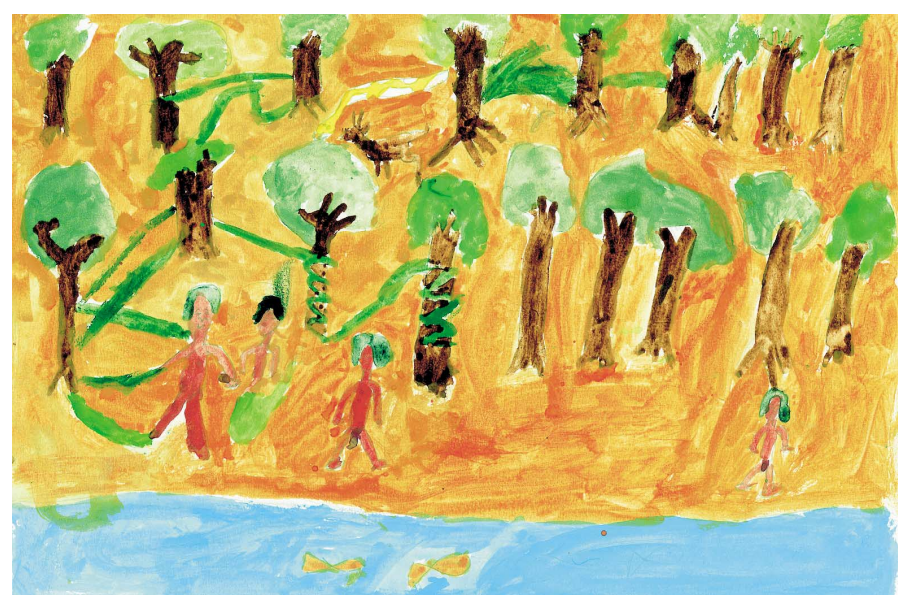

\section{Figure 6}

La liane hali hali descendue à terre, se transforme en jeune Amérindien puis s'intègre au groupe en y prenant femme afin de se révéler à ces derniers. Dessin de Bioman, jeune écolier de 11 ans, Classe de CM1, Antecume Pata, Guyane française, octobre 2000.

\section{Figure 6}

The hali hali vine becomes a young Wayana Indian after having come down to earth. He integrates himself into the group by taking a wife; and, thus, reveals to the group the secret of the vine. Drawing from Bioman, a 11 year old pupil, CM1 level, Antecume Pata, French Guyana, October 2000.

Qu'elles soient villageoises ou commerciales, les grandes nivrées garantissent une production abondante en kumaru, particulièrement celles à but commercial, organisées de façon semi clandestine par un nombre réduit de participants qui se sont cooptés. La grande nivrée commerciale à laquelle nous avons assisté sur l'Oelemari en octobre 1998 a produit 58 kumaru que se sont répartis les 8 pêcheurs adultes (Tableau I).

\section{Tableau I}

Eléments de comparaison entre deux types de grandes nivrées, l'une commerciale (Kilipirialu, Oelemary, octobre 1998), l'autre villageoise (Saut Pierkuru, Tampok, octobre 2000). Sources JÉGU et PAGEZY, 1999 et Opération Nivrée 2000 ( ${ }^{*}$ sur 17 pirogues).

Table I

Comparison of two kinds of fishing parties of major importance: one is commercial (Kilipirialu, Oelemary, October 1998), the other one concerns a whole village (Saut Pierkuru, Tampok, October 2000). From JÉGU and PAGEZY (1999) and the "Nivrée 2000 " research program (unp. data) (" observations from 17 pirogues).

\begin{tabular}{|c|c|c|c|c|c|}
\hline & $\begin{array}{c}\text { Nombre de } \\
\text { pirogues }\end{array}$ & $\begin{array}{c}\text { Nombre de } \\
\text { pêcheurs }\end{array}$ & $\begin{array}{c}\text { Nombre de. fagots } \\
\text { (poids moyen) } \\
\text { Poids total }(\mathrm{kg})\end{array}$ & $\begin{array}{c}\text { Nombre de } \\
\text { kumaru / pirogue }\end{array}$ & $\begin{array}{c}\text { Nombre de. } \\
\text { kumaru / pêcheur }\end{array}$ \\
\hline $\begin{array}{c}\text { Nivrée } \\
\text { commerciale }\end{array}$ & 5 & 8 & $\begin{array}{c}51(8,4 \mathrm{~kg}) \\
429 \mathrm{~kg}\end{array}$ & $11(7-22)$ & 5 à $7(1$ fois 15) \\
\hline $\begin{array}{c}\text { Nivrée } \\
\text { villageoise }\end{array}$ & 24 & 48 & $\begin{array}{c}72(10,4 \mathrm{~kg}) \\
750 \mathrm{~kg}\end{array}$ & $23(5-56)^{\star}$ & 3 à $28^{*}$ \\
\hline
\end{tabular}


Les petites nivrées ou celles d'importance moyenne peuvent impliquer une personne, une famille voire un village, comme à Antecume Pata dont le caractère particulier d'île entourée de rapides apparaît favorable. Ces pêches ont des cibles beaucoup plus diversifiées, les Serrasalminae étant dans ce cas surtout représentés par le laku (Acnodon). II n'en reste pas moins vrai que certaines espèces ou certaines familles de poisson y sont plus particulièrement recherchées, comme les Loricariidae ${ }^{9}$ dans les nivrées pratiquées en eau vive ou les Callichthyidae représentés par l'etpë (Callichtys callichtys), espèce cible des petites nivrées familiales de boue (JÉGU et PAGEZY, 1999).

II n'en reste pas moins vrai que la fonction essentielle des nivrées villageoises est sociale et éducative : l'expérience de la vie communautaire autour d'un grand rassemblement des membres de la communauté amérindienne ayant comme effet le renforcement de la cohésion du groupe. II s'agit d'un événement que personne ne manquerait ${ }^{10}$. Ainsi, au jour annoncé par le chef de nivrée, la famille part au grand complet, par vagues de pirogues apparentées, pleines à craquer. L'ambiance de la nivrée est particulièrement conviviale : on mange bien, on s'entraide, on partage la nourriture au sein de la famille, on dort dans des carbets improvisés, au contact avec la nature, on parle tard le soir.

Dès leur plus jeune âge, les enfants sont culturellement enracinés au cours de ces grandes expéditions : dès l'âge de 5 ans, les jeunes Indiens s'exercent à pêcher à la flèche-harpon depuis la rive ou attrapent les petits poissons à la main ${ }^{11}$. Ils observent, apprennent et vivent l'expérience de leurs aînés : la façon dont ceux-ci choisissent leur itinéraire, dont ils viennent à bout des difficultés générées par le passage dans les rapides, dont ils choisissent l'emplacement des bivouacs, dont ils pêchent le soir à l'épervier, au filet ou à la ligne.

L'investissement de la population toute entière, en temps, en travail et en argent sur un projet commun illustre bien la valeur sociale que représente un tel événement. La recherche des lianes ${ }^{12}$ à 2 ou 3 reprises dans la semaine qui précède l'opération (une ultime campagne pouvant avoir lieu sur le chemin) mobilise chaque fois une journée complète de 3 hommes ou plus ; au cours d'une telle opération, il faut grimper dans l'arbre qui sert de support à la liane, l'abattre et transporter le lourd butin dans les pirogues.

De leur côté, les femmes préparent la nourriture en abondance : galettes de manioc d'un mètre de diamètre environ (5 à 10 par pirogue), farine de manioc (15 à $20 \mathrm{~kg}$ ), sauce pimentée, bière de manioc.

II faut aussi penser au carburant (100 à 200 litres d'essence, huile de moteur), acheter les cartouches, les pains de glace et le sel pour conserver le poisson, acheter quelques provisions complémentaires : riz, sucre, café, thé, huile.

Les instruments de pêche doivent être vérifiés et les membres de la famille encore éparpillés doivent rapidement se rassembler pour le grand départ.

\footnotetext{
9 Pële (Pseudancistrus barbatus), pële simali (Ancistrus spp.), mili (Hemiancistrus medians), kawawa (Hypostomus spp. et Ancistrinus temminckii), lapipi (Harttia spp., Farlowella spp., Metaloricaria spp.), meikolo (Pseudancistrus serratus).

${ }^{10}$ Au village de Cayodé, lors de la grande nivrée d'octobre 2000, ne sont restées que 5 personnes sur 123, dont 3 personnes âgées.

11 «A partir de 11 ans, la pêche devient l'activité principale des garçons » chez les Wayãpi selon ROMEU (2000). Au cours d'une petite nivrée, la mère d'un enfant d'environ 3 ans envoyait des petits poissons à son bébé qui, assis au bord de l'eau, s'appliquait à les percer de sa flèche, jusqu'à ce qu'un nouveau lui parvienne (opération « Nivrée $2000 »)$.

12 Les informations qui suivent proviennent essentiellement de la grande nivrée villageoise d'octobre 2000 au saut Pierkuru sur le Tampok, mais aussi de petites nivrées et d'une nivrée commerciale.
} 
L'importance de la durée du transport jusqu'au lieu de pêche, de une à trois journées de pirogue, le passage de nombreux sauts par des pirogues très chargées, impliquent de la part des participants de nombreuses qualités d'adresse, de persévérance, d'aide mutuelle ; ce sont autant d'indicateurs de cet investissement collectif en vue d'une activité hautement valorisée par la société.

Le battage des lianes la veille de l'opération au moyen de lourds gourdins, tous les hommes se relayant pour écraser sans répit les centaines de $\mathrm{kg}$ de végétaux durant 5 à 8 heures, révèle encore l'investissement collectif occasionné par ce type de pêche.

\section{La pêche à la ligne appâtée par des fruits}

En saison des pluies se pratique une pêche très particulière qui met en jeu les connaissances ethnobiologiques du milieu et des relations entre les végétaux et les animaux. II s'agit d'une pêche à la ligne dont l'hameçon est appâté de fruits ${ }^{13}$ dont raffolent les Serrasalminae herbivores, comme les kumaru et le pasina (cf. OUHOUD-RENOUX, 1998 pour l'Oyapok). Assis dans son canot, le pêcheur lance sa canne d'un coup sec de part et d'autre du bateau en se laissant dériver d'amont en aval le long des rives vers les sauts, tout en frappant l'eau de sa pagaie pour imiter le fruit qui tombe dans l'eau. Pierre Grenand (1993) a qualifié la stratégie développée à l'occasion de cette pêche d'« optimisation simple ».

Plus particulièrement en saison des pluies, certains Indiens partent durant plusieurs jours pour des campagnes à but strictement commercial. Ils remontent le fleuve durant 3 à 5 jours (le temps pour les pains de glace de rester efficaces), associant chasse et pêche, de nuit comme de jour.

\section{éducative}

Les dessins d'enfants, révélateurs d'une activité emblématique à forte portée

Qualifier de patrimoniales les principales techniques de pêche aux kumaru sousentend qu'elles soient transmises aux jeunes générations (CORMIER-SALEM et ROUSSEL, 2000).

Dans le cas des grandes nivrées et de la pêche aux watau avec appâts spécifiques, il semble bien que les enfants aient reçu de façon précoce la transmission de savoirs et de techniques spécifiques. C'est ce qu'il ressort des attitudes des personnages et des détails relevés dans les dessins d'écoliers âgés de 7 à 15 ans et dans leurs récits de pêche ${ }^{14}$. Leurs connaissances de la biologie de ces poissons, de leur habitat privilégié, de leur nourriture favorite, et des outils de pêche utilisés semblent, déjà à 7 ans, pratiquement fixées et stabilisées.

En ce qui concerne la pêche à la nivrée, l'action se situe pour la plupart des dessins au niveau des sauts (Figure 7.1). Plusieurs canots descendent le courant tandis que des pêcheurs se déplacent sur les rochers. Ceux ci sont recouverts de Podostemaceae wija (Mourera fluviatilis Aubl.), que l'on reconnaît par leur couleur

\footnotetext{
${ }^{13}$ Nous avons relevé (JÉGU et PAGEZY, 1999) les appâts suivant pour les kumaru : aluti (ind), elekesi (Macrolobium acaciifolium (Benth.) Benth. Caesalpiniaceae), enayop (Croton cuneatus, Klotz., Euphorbiaceae), konoi (Gurania spinulosa (Poep. et Endl.) Cogn. Cucurbitaceae), kupayuwa (Copaifera guianensis Desf., Caesalpiniaceae), kutoyu (Passiflora sp., Passifloraceae), makolomuna (ind), mope (Spondias mombin L. Anacardiaceae), pawe (Peltogyne paniculata Benth. Caesalpiniaceae), pisusuk (Genipa spruceana Steyerm. Rubiaceae), sihkëimë (Henriettea cf stellaris O. Berg ex Triana, Melastomataceae), ulimë (cf Cayaponia sp. Cucurbitaceae), wapa (Eperca falcata Aubl. Caesalpiniaceae).

${ }^{14} \mathrm{Au}$ cours de la mission de saison des pluies (mai 1999) nous avons pu travailler avec les enfants des écoles primaires de Pidima (CP-CM2 ; 7 à 11 ans) et d'Antecume Pata (CE2-CM2 ; 9 à 15 ans), grâce à la collaboration des instituteurs. II a été proposé aux enfants de dessiner ou peindre une pêche au kumaru. A Antecume, les enfants les plus âgés ont accompagné leur dessin d'une rédaction (JÉGU et PAGEZY, 1999).
} 
verte, exhibant parfois leurs magnifiques fleurs roses (Figure 7.2). Certains enfants ont représenté le battage des lianes avant la nivrée (Figure 7.3), ou des pêcheurs en train de flécher un kumaru avec leur arc (Figure 7.4). La force de l'animal et l'effort du pêcheur apparaissent d'une façon très réaliste : arc-boutés sur leur canne à pêche, certains ont du mal à tirer le poisson hors de l'eau. Ricardo (11 ans, CM1), quant à lui, a représenté les diverses phases d'une pêche à laquelle il a participé avec son père, depuis le départ du groupe vers le "saut » jusqu'à la vente des kumaru à Maripasoula puis le retour au village avec le fût d'essence acheté grâce à la vente.

Les enfants ont aussi représenté des pêches aux kumaru que l'on pratique en saison des pluies, au moyen de lignes appâtées de Podostemaceae wïja ou de fruits jaune orangé (Figure 7.5 et 7.6), comme pisusuk ou mope (cf. note 11). Assis dans sa pirogue, le pêcheur lance sa canne de part et d'autre du canot. II la tient d'une main ferme, tandis que de l'autre il dirige son embarcation. Les watau arrivent nombreux, attirés par le parfum exhalé par les wijja ou les fruits dont ils raffolent. La ligne se courbe sous la tension du poisson. Le pêcheur remonte la canne d'un geste rapide. D'un coup de foëne, gardée à portée de main, il embroche le poisson qu'il a préalablement assommé ou bien il le flèche avec son arc. La grande force du kumaru comme l'adresse du pêcheur ressortent aussi des récits.

\section{Valeur économique des kumaru}

Selon CORMIER SALEM (2000), le patrimoine vivant est éphémère, susceptible de se transformer, de s'altérer ou de s'enrichir. Les choix effectués par les groupes sociaux, notamment en fonction de leurs systèmes de pensée, peuvent aussi évoluer. Ainsi les pratiques innovantes, comme la pêche semi-commerciale ou commerciale, pourront dans l'avenir se traduire localement par des modifications de la biodiversité.

La chasse et la pêche représentent pour certaines familles les principales activités lucratives leur permettant de faire face aux dépenses courantes. Dans le haut Maroni, la vente d'animaux sauvages se fait selon 3 modalités : dans les villages à des Indiens sur place (surtout à l'occasion de fêtes) ou à Maripasoula soit à des consommateurs, soit à des revendeurs boni.

L'importance des kumaru aux yeux des Boni se manifeste par le fait que, sitôt qu'une pirogue approche de l'embarcadère, les clients se précipitent en lançant des objets dans le canot afin de se les réserver. Le prix au $\mathrm{kg}$ atteint par les kumaru ${ }^{15}$, qui n'est égalé que par les poissons roches Loricariidae, rend aussi compte de la valeur que ces espèces représentent aux yeux des Noirs marrons. Après une grande nivrée, le bénéfice de la vente d'une dizaine de poissons peut atteindre $229 €$ à $305 €$, auquel s'ajoute les revenus de la chasse. Cela place ces espèces en bonne position dans les sources de revenus des Indiens, bien qu'elles ne soient pas les seules à être commercialisées. Au cours des mêmes campagnes, les poissons roche, les aimara, des huluwi, les œufs d'iguane et les gibiers comme le maipuri pouvant atteindre $60 \mathrm{~kg}$, sont facilement vendues. Pour certains pêcheurs, les grandes nivrées peuvent assurer des revenus substantiels (plus de $610 €$, voire $762 €$ à $1220 €$ ), les kumaru représentant dans ce cas l'essentiel des bénéfices.

Depuis peu, la pratique commerciale de la pêche s'est développée, en relation avec les besoins croissants des populations amérindiennes en articles importés : savon, lessive, farine, café, sucre, habits, frais de scolarité. Mais c'est l'essence, qui reste le bien de consommation indispensable, lié à la possession quasi unanime par les pêcheurs de moteurs hors-bord tractant les pirogues. Le fait que l'essence apparaisse comme la « monnaie d'échange » la plus appréciée nous semble un indicateur de sa haute valeur socio-économique (JÉGU et PAGEZY, 1999).

${ }^{15} 6,1 € / \mathrm{kg}$ en octobre $1998,7,6 € / \mathrm{kg}$ en octobre 2000 , soit 22,9 à $38,1 €$ l'unité. 

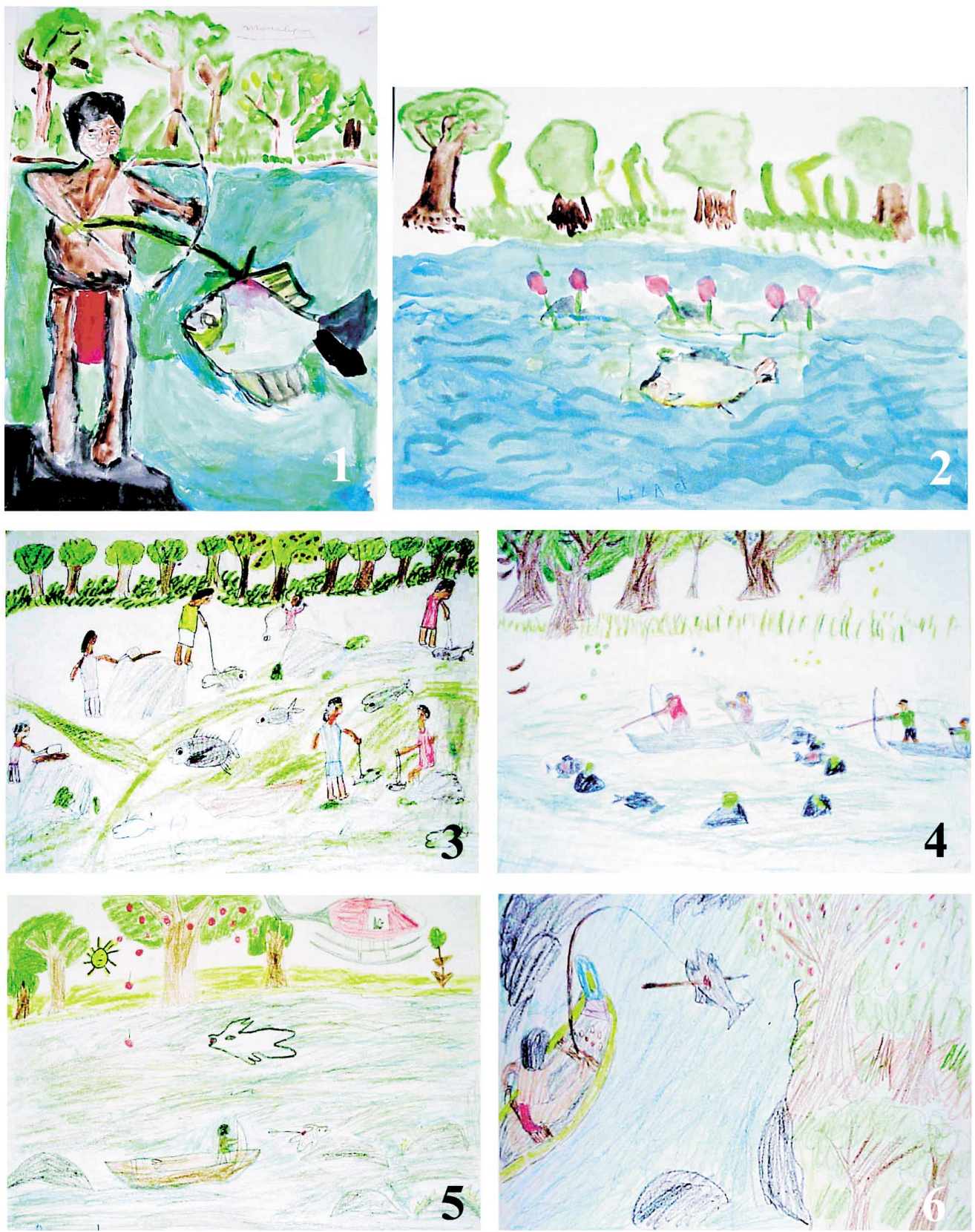

\section{Figure 7}

Représentation des pêches aux kumaru par les enfants des écoles primaires de Pidima et Antecume Pata, Guyane française : 1 - dessin de Monalipo, garçon de 11 ans ; 2 - dessin de Kela, fille de 9 ans ; 3 - dessin d'Hameleu, fille de 10 ans ; 4 - dessin de Maurice, garçon de 8 ans ; 5 - dessin de Maiki, garçon de 13 ans ; 6 - dessin de Reymond, garçon de 10 ans.

\section{Figure 7}

Representation of kumaru fishing parties drawn by primary schools children of Pidima and Antecume Pata, French Guyana: 1 - drawing of Monalipo, a 11 year old boy; 2 - drawing of Kela, a 9 year old girl; 3 - drawing of d'Hameleu, a 10 year old girl; 4 - drawing of Maurice, a 8 year old boy; 5 - drawing of Maiki, a 13 year old boy; 6 - drawing of Reymond a 10 year old boy. 
Le passage actuel des Wayana d'une économie de subsistance à une économie de marché reposant sur l'argent, répond à de nouvelles aspirations touchant essentiellement une meilleure qualité de vie et suscite de nouveaux besoins, ayant pour conséquence une demande accrue de kumaru destinés à la commercialisation. La logique de profit (vente) qui s'oppose à la logique de subsistance (autoconsommation, partage), peut dans un premier temps coexister pour ensuite entrer en conflit à l'issue duquel l'une cèdera la place à l'autre. La commercialisation d'une partie de la production de pêche ou de chasse est vitale pour rentrer dans les frais occasionnés par des campagnes de pêche (essence, provisions, pains de glace, etc.), l'opération n'étant pas toujours garantie en cas d'incident coûteux comme une hélice endommagée.

\section{Valeur d'existence des kumaru}

En 1968, le concept de valeur d'existence (« existence value ») est apparu chez les économistes américains (KRUTILLA, 1967). II repose sur une méthode d'évaluation contingente, consistant à chiffrer la valeur d'une espèce ou d'un milieu par le biais du montant maximal que serait disposée à payer une population afin de conserver ces derniers en l'état (VIVIEN, 2000). Cette notion est-elle transposable aux kumaru dans le contexte du haut Maroni ? A-t-elle seulement un sens pour les sociétés amérindiennes encore peu monétarisées?

Transmettre aux générations futures les valeurs sociales, culturelles, économiques, que représentent les kumaru et leurs modes d'appropriations ne pourra se réaliser que si la ressource est gérée à long terme. Selon BADY (1995), l'appartenance à un patrimoine implique " la nécessité d'une forme d'attachement de la part d'une collectivité ou d'une communauté (...) Une marque collective traduisant un intérêt privilégié est indispensable ». C'est ainsi qu'une prise de conscience collective sur une possible diminution des stocks dans le Haut Maroni a incité, il y a quelques années, le grand Man des Wayana à interdire la pêche à la nivrée avec l'accord de la population ${ }^{16}$. Ce changement de comportement estil si éloigné de la déclaration d'intention proposée par les économistes pour évaluer la valeur d'existence d'une espèce dans des pays occidentaux ? Pour les sociétés peu monétarisées, renoncer aux bénéfices sociaux, économiques et culturels de la ressource, en changeant de comportement dans le but de préserver celle-ci, est déjà lui accorder une valeur d'existence. La transposition chiffrée est certes plus délicate, mais une amorce de réponse peut être apportée par l'estimation du manque à gagner généré par le déficit de production.

Le fait que cette prise de décision n'ait pas tenu longtemps est vraisemblablement le signe d'un conflit entre deux logiques en présence, la logique ancestrale de subsistance s'opposant à la logique moderne de profit.

\section{CONCLUSION GÉNÉRALE}

Du point de vue du biologiste, les trois espèces de kumaru du Haut Maroni se situent à des niveaux d'endémicité différents, mais toutes participent au fonctionnement du biotope particulier des herbiers à Podostemaceae, au moins dans leurs jeunes stades. Audelà de la simple conservation des espèces, le mode de distribution de l'asitau et la position du watau yaikë dans la phylogénie des Serrasalminae leur confèrent un ensemble de qualités entrant dans l'évaluation de leur valeur patrimoniale. L'habitat de ces espèces, milieu fragile et très particulier, participe aussi à la patrimonialité.

Du point de vue de l'anthropologue, les kumaru apparaissent indéniablement comme les espèces de poissons ayant globalement les plus fortes valeurs sociale,

${ }^{16}$ Jean CHAPUIS (comm. pers.). 
hédonique, économique, non seulement chez les Amérindiens Wayana du Haut Maroni, mais aussi chez les Noirs Boni du cours moyen. On peut les qualifier à ce titre d'espèces patrimoniales. Ces espèces emblématiques du haut Maroni, méritent bien la qualification de patrimoniales, et ce à plusieurs titres, débordant largement leur valeur intrinsèque culturelle et économique ; leurs modes de production, transmises aux jeunes générations, ont dans le cas de la pêche à la nivrée une valeur identitaire.

Espèces particulièrement appréciées, les kumaru sont aussi des espèces faiblement contaminées par le mercure (FRÉRY et al., 1999) au même titre que les poissons roches. On peut regretter que parmi les poissons susceptibles de procurer des revenus, les grands carnivores comme l'aimara et le poisson tigre huluwi présentent des teneurs en mercure élevées. A l'inverse, la faible contamination des kumaru risque de renforcer non seulement leur valeur d'existence mais aussi leur valeur marchande et inciter à la vente.

La pression sur les kumaru ne cesse de progresser, aussi bien à cause des changements récents dans le mode de vie des Wayana que des modifications du milieu induites par l'activité d'orpaillage. Cette pression est susceptible de s'accroître dans la mesure où la contamination par le mercure affecte essentiellement d'autres espèces carnivores présentes dans l'alimentation, ce qui pourrait conduire les populations locales à reporter leurs efforts de pêche, en vue de la consommation ou de la vente, sur des espèces moins contaminées que sont les kumaru.

\section{REMERCIEMENTS}

Ce travail a été réalisé dans le cadre du Programme "Impacts Ecologiques des Activités Humaines traditionnelles sur l'Ecosystème Forestier Guyanais » du M.A.T.E., Convention IRD / ECOFOR 2350, du Programme « Ecosystèmes Tropicaux » du M.A.T.E., Convention IRD / ECOFOR 2498 et de la Convention IRD / CSP 2548. Nous remercions les populations amérindiennes du Haut Maroni ainsi que les membres de l'opération de recherche « Nivrée 2000 »: S. CARRIĖRE (IRD, Bondy), B. FAUJOUR (ENSAT, Toulouse), Y. FERMON (MNHN, Paris), O. FOSSATI (IRD, Lyon), P. KEITH (MNHN, Paris), P.Y. LE BAIL (INRA, Rennes), F. MEUNIER (MNHN, Paris), S. MULLER (MHNG, Genève), P. SALAÜN (Mission Parc, Cayenne) et C. WEBER (MHNG, Genève). Nous remercions aussi Philippe KEITH et Bernard ROUSSEL (Museum National d'Histoire Naturelle) pour leurs précieux commentaires.

\section{BIBLIOGRAPHIE}

BADY, 1995. Ouverture du colloque. In : Patrimoine culturel, patrimoine naturel, Actes du colloque des 12 et 13 décembre 1994. Ecole Nationale du Patrimoine, Paris : La Documentation Française, p. 12.

BOUJARD T., SABATIER D., ROJAS-BELTRAN R., PRÉVOST M.F., RENNO J.F., 1990. The foods habits of three allochthonous feeding characoids in French Guiana. Rev. Ecol. (Terre et Vie), 45, 247-258.

BOUTHELOUP E., 1999. Une espèce peut-elle être patrimoniale ? Rapport de stage de recherche documentaire en sciences naturelles, Paris, juillet 1999, $42 \mathrm{p}$.

CHAPUIS J., 1998. La personne Wayana entre sang et ciel. Thèse de Doctorat, Université. d'Aix Marseille III, $1006 \mathrm{p}$.

CORMIER-SALEM M.C., ROUSSEL B., 2000. Patrimoines naturels : La surenchère. La Recherche, 333, 105-110.

CORMIER-SALEM M.C., 2000. Les stratégies locales dans la construction de patrimoines naturels et de territoires. Projet scientifique, Unité de Recherche IRD. 
DIREN, 1998. Liste des espèces patrimoniales, Inventaire Znieff : Notice fiche espèces patrimoniales. Comité Scientifique Régional du Patrimoine Naturel Guyanais, 06 janvier 1998. 3 pages ronéo.

FERREIRA E.G., 1992. A Ictiofauna do rio Tombetas na Area de Influência da futura usina hidrelétrica de Cachoeira Porteira, Pará. Tese de doutorada, FUA, Manaus, 160 p.

FISCHLER C., 1989. L'Homnivore. Paris : Odile Jacob, 414 p.

FRÉRY N., MAILLOT E., DEHEEGER M., 1999. Exposition au mercure de la population amériendienne Wayana de Guyane. Enquête alimentaire. Institut de Veille Sanitaire, juin 1999, $82 \mathrm{p}$.

GOULDING M., CARVALHO M.L., FERREIRA E.G., 1988. Rio Negro: Rich life in poor Water: Amazonian Diversity and Foodchain Ecology as seen Through Fish Communities. The Hague, SPB Academic Publishing.

GRENAND P., 1996. Des fruits, des animaux et des hommes. Stratégies de la chasse et de la pêche chez les Wayãpi d'Amazonie. In: HLADIK C.M. et al. (Eds.), L'alimentation en forêt tropicale : interactions bioculturelles et applications au développement. UNESCO/MAB, collection Man and Biosphere, 671-684.

GRENAND P., MORETTI C., 1982. Les nivrées ou plantes ichtyotoxiques de la Guyane française. J. of Ethnopharmacology, 6, 139-160.

HOFF M., 2000. Liste des habitats de Guyane. Conseil Scientifique Régional du Patrimoine Naturel de Guyane. Compte-rendu des rencontres des 3 et 4 février 2000, DIREN, $3 \mathrm{p}$.

HURAULT J., 1963. Chasse et pêche chez les populations d'origine africaine en Guyane. Bois et forêts des tropiques, 87, 3-8.

JANVIER P., 1999. Coelacanth à la Marseillaise. Nature, 401, 854-856.

JÉGU M., KEITH P., BELMONT-JÉGU E., 2002. Une nouvelle espèce de Tometes (Teleostei : Characidae : Serrasalminae) du bouclier guyanais, Tometes lebaili n. sp. Bull. Fr. Pêche Piscic., 364, 23-48.

JÉGU M., PAGEZY H., 1999. Ecologie des kumaru et étude d'une production traditionnelle des hauts cours des fleuves guyanais. Rapport de fin de contrat, Convention IRD ECOFOR $\mathrm{n}^{\circ} 2350,67$ p. et $8 \mathrm{pl}$.

KEITH P., LE BAIL P.Y., MÉRONA B. De, 2000. Espèces patrimoniales de poissons d'eau douce de Guyane. Conseil Scientifique Régional du Patrimoine Naturel de Guyane, Compte-rendu des rencontres des 3 et 4 février 2000, DIREN, 3 p.

KRUTILLA J.V., 1967. Conservation Reconsidered. The American Economic rewiew, 57, 777-786.

LE BAIL P.Y., KEITH P., PLANQUETTE P., 2000. Atlas des poissons d'eau douce de Guyane. Tome 2, fascicule II. Siluriformes. Patrimoines naturels (M.N.H.N., S.P.N.), 43 (2), $307 \mathrm{p}$.

LEVI STRAUSS C., 1962. Le Totemisme Aujourd'hui. Paris, PUF, $154 \mathrm{p}$.

LECOMTE F., BOUJARD T., MEUNIER F.J., RENNO J.F., ROJAS-BELTRAN R., 1993. The growth of Myleus rhomboidalis (Cuvier, 1817) (Characiformes, Serrasalmidae) in two rivers of French Guiana. Rev. Ecol. (Terre Vie), 48, 421-435.

MEUNIER F, KEITH P., LE BAIL P.Y., 1998. Conserver les spécificités de l'ichtyofaune guyanaise. JATBA, revue d'Ethnobiologie, 40 (1-2), 239-259.

MYERS A.A., DE GRAVE S., 2000. Endemism: Origins and implications. Vie et Milieu, 50 (4), 195-204.

ODINETZ-COLLART O., JÉGU M., THATCHER V.E., TAVARES A.S., 1996. Les prairies aquatiques de l'Amazonie brésilienne. ORSTOM Actualités, 49, 8-14.

ORTI G., PETRY P., PORTO J.I.R., JÉGU M., MEYER A., 1996. Patterns of Nucleotide Change in Mitochondrial Ribosomal RNA gene and the Phylogeny of Piranhas. J. Mol. Evol., 42, 169-182.

OUHOUD-RENOUX F., 1998. De l'outil à la prédation. Technologie culturelle et ethnoécologie chez les Wayãpi du haut Oyapock (Guyane française). Thèse présentée en vue du grade de Docteur à l'Université de Paris X, 455 p. 
PORTO J.I., FELDBERG E., NAKAYAMA C., JÉGU M., 1991. Cytotaxonomic analysis in the Serrasalmidae Ostariophysi, Characiformes). VII Congress of European Ichthyology, Bull. Zool. Mus. Univ. Amsterdam, The Hague.

PLANQUETTE P., KEITH P., LE BAIL P.Y., 1996. Atlas des poissons d'eau douce de Guyane (tome 1). Collection du Patrimoine Naturel, vol 22, IEGB - MNHN, INRA, CSP, Min. Env., Paris, 214 p.

ROMEU S., 2000. L'enfant Wayãpi et la rivière. Exploitation des ressources naturelles accessibles par le cours d'eau. Mémoire de DEA Environnement : temps, espace, société, Université d'Orléans, $165 \mathrm{p}$.

SANTOS G.M. Dos, JÉGU M., MÉRONA B. De, 1984. Catalogo dos peixes commerciais do Baixo Tocantins. Eletronorte, Brasilia, 84 p.

SANTOS G.M., PINTO S.S., JÉGU M., 1997. Alimentação do pacu-cana Mylesinus paraschomburgkii (Teleostei, Serrasalmidae) em rios da Amazônia brasileira. Rev. Brasil. Biol., 57(2), 311-315.

THATCHER V.E., JÉGU M., 1996. Intestinal helminths as population markers of the Amazonian fish Mylesinus paraschomburgkii, with description of five new genera and seven species of trematodes. Amazoniana, 14 (1/2), 143-155.

VIVIEN F.D., 2000. Quel prix accorder à la biodiversité. La Recherche, 333, 88-91. 
